\title{
Diffusion Approximations in the Online Increasing Subsequence Problem
}

\author{
Alexander Gnedin and Amirlan Seksenbayev
}

January 9, 2020

\begin{abstract}
The online increasing subsequence problem is a stochastic optimisation task with the objective to maximise the expected length of subsequence chosen from a random series by means of a nonanticipating decision strategy. We study the structure of optimal and near-optimal subsequences in a standardised planar Poisson framework. Following a long-standing suggestion by Bruss and Delbaen [8], we prove a joint functional limit theorem for the transversal fluctuations about the diagonal of the running maximum and the length processes. The limit is identified explicitly with a Gaussian time-inhomogeneous diffusion. In particular, the running maximum converges to a Brownian bridge, and the length process has another explicit non-Markovian limit.
\end{abstract}

\section{Introduction}

We adopt the following standardised framework for the online stochastic optimisation problem of Samuels and Steele [21. Suppose i.i.d. marks drawn from the uniform distribution on $[0,1]$ are observed, one by one, at times of independent homogeneous Poisson process of intensity $\nu$ on $[0,1]$. Each mark can be selected or rejected. The sequence of selected marks must increase. The task is to maximise the expected length of selected increasing subsequence using an online strategy. The online constraint requires that each decision becomes immediately irrevocable as the mark is observed and must be based exclusively on the information accumulated previously without foresight of the future.

The optimal online strategy is defined recursively in terms of a variable acceptance window, which limits the difference between the next and previous selections. The strategy and its value can be found, in principle, by solving a dynamic programming equation, see [4, 7, 14 for properties of the solution and approximations. Our main theme is different. We study the time evolution of increasing subsequences under online strategies that are within $O(1)$ gap from the optimum for large $\nu$.

Let $L_{\nu}(t)$ and $X_{\nu}(t)$, respectively, denote the length and the last element of the increasing subsequence selected by time $t \in[0,1]$ under the optimal strategy. The interest to date focused on the total length $L_{\nu}(1)$. Samuels and Steele derived the principal asymptotics $\mathbb{E} L_{\nu}(1) \sim \sqrt{2 \nu}$, which was later found to be an upper bound with the optimality 
gap of order $\log \nu$ [7]. See [1, 2, 4, 7, 13] for refinements and generalisations. In a recent paper [14] we combined asymptotic analysis of the dynamic programming equation with a renewal approximation to the range of the process $Z_{\nu}(t):=\sqrt{\nu(1-t)\left(1-X_{\nu}(t)\right)}$ to derive expansions for the mean

$$
\mathbb{E} L_{\nu}(1) \sim \sqrt{2 \nu}-\frac{1}{12} \log \nu+c_{0}^{*}, \quad \nu \rightarrow \infty,
$$

and the variance

$$
\operatorname{Var} L_{\nu}(1) \sim \frac{1}{3} \sqrt{2 \nu}-\frac{1}{72} \log \nu+c_{1}^{*}, \quad \nu \rightarrow \infty,
$$

where $c_{0}^{*}$ and $c_{1}^{*}$ are unknown constants. A central limit theorem for $L_{\nu}(1)$ was proved in [8] by analysis of a related martingale, and further extended in [14] to a larger class of asymptotically optimal strategies by the mentioned renewal theory approach.

Samuels and Steele introduced the online selection problem as an offspring of the muchstudied Ulam-Hammersley problem on the longest increasing subsequence of the Poisson scatter of points in the square $[0,1]^{2}$. They viewed the Ulam-Hammersley problem as an offline optimisation task with the complete foresight of choosable options. In the offline framework, the well known principal asymptotics of the expected maximum length, $2 \sqrt{\nu}$, is similar, but the second term of its asymptotic expansion and the principal term of the standard deviation are both of the order $\nu^{1 / 6}$. The limit law for the offline maximum length is the Tracy-Widom distribution from the random matrix theory. An excellent reference here with an exposition of the history is the book by Romik [20].

In the offline problem, some work has been done on the size of transversal fluctuations about the diagonal $x=t$ in $[0,1]^{2}$. Johansson [17] proved a measure concentration result asserting that, with probability approaching 1 , every longest increasing subsequence (which is not unique) lies in a diagonal strip of width of the order $\nu^{-1 / 6+\epsilon}$. Duvergne, Nica and Virág [11] recently proved the existence and gave some description of the functional limit, which is not Gaussian. But for smaller exponent $-1 / 2<\alpha<-1 / 6$, Joseph and Peled [12] showed that if the increasing sequence is restricted to lie within the strip of width $\nu^{-\alpha}$, the expected maximum length remains to be asymptotic to $2 \sqrt{\nu}$, while the limit distribution of the length switches to normal.

To extend the parallels and gain further insight into the optimal selection it is of considerable interest to examine fluctuations of the processes $L_{\nu}$ and $X_{\nu}$ as a whole. On this path, one is lead to study the following scaled and centred versions of the running maximum and length processes:

$$
\widetilde{X}_{\nu}(t):=\nu^{1 / 4}\left(X_{\nu}(t)-t\right), \quad \widetilde{L}_{\nu}(t)=\nu^{1 / 4}\left(\frac{L_{\nu}(t)}{\sqrt{2 \nu}}-t\right), \quad t \in[0,1] .
$$

To compare, in the offline problem by similar centring the critical transversal and longitudinal scaling factors appear to be $\nu^{1 / 6}$ and $\nu^{1 / 3}$, respectively. Our central result (Theorem 40 is a functional limit theorem which entails that the process $\left(\widetilde{X}_{\nu}, \widetilde{L}_{\nu}\right)$ converges weakly to a simple two-dimensional Gaussian diffusion. In particular, $\widetilde{X}_{\nu}$ approaches a Brownian bridge. The limit of $\widetilde{L}_{\nu}$ is a non-Markovian process with the covariance function

$$
(s, t) \mapsto \frac{2 s(2-t)-(2-s-t) \log (1-s)}{6 \sqrt{2}}, \quad 0 \leq s \leq t \leq 1,
$$


which corresponds to a correlated sum of a Brownian motion and a Brownian bridge,

The question about functional limits for $L_{\nu}$ and $X_{\nu}$ has been initiated by Bruss and Delbaen [8]. They employed the Doob-Meyer decomposition to compensate the processes, and in an analytic tour de force showed that the scaled martingales jointly converge to a correlated Brownian motion in two dimensions. However, the compensation keeps out of sight a drift component absorbing much of the fluctuations immanent to the selection process, let alone that the compensators themselves are nonlinear integral transforms of $X_{\nu}$. To break the vicious circle one needs to obtain the limit of $X_{\nu}$ under complete control over the centring. Curiously, in the forerunning paper Bruss and Delbaen mentioned that P.A. Meyer had suggested to them to scrutinise the generator of the Markov process $\left(X_{\nu}, L_{\nu}\right)$ (see [7], Remark 2.4).

Looking at the generator of (3) we shall recognise the limit process without difficulty. But in order to justify the weak convergence in the Skorokhod space on the closed interval $[0,1]$ we will need to circumvent a difficulty caused by pole singularities of the control function and the drift coefficient at the right endpoint. We shall also discuss related processes and derive tight uniform bounds on the expected values of $X_{\nu}$ and $L_{\nu}$, thus embedding the moment expansions from [14] in the functional context.

Notation. We sometimes omit dependence on the intensity parameter $\nu$ wherever there is no ambiguity. Notation $X$ and $L$ will be context-dependent, typically standing

for processes associated with a near-optimal online selection strategy, while $\widetilde{X}$ and $\widetilde{L}$ will denote the normalised versions with scaling and centring as in (3).

\section{Selection strategies}

It will be convenient to extend the underlying framework slightly by considering a homogeneous Poisson random measure $\Pi$ with intensity $\nu$ in the halfplane $\mathbb{R}_{+} \times \mathbb{R}$, along with the filtration induced by restricting $\Pi$ to $[0, t] \times \mathbb{R}$ for $t \geq 0$. We interpret the generic atom $(t, x)$ of $\Pi$ as random mark $x$ observed at time $t$. A sequence $\left(t_{1}, x_{1}\right), \ldots,\left(t_{\ell}, x_{\ell}\right)$ of atoms is said to be increasing if it is a chain in two dimensions, i.e.

$$
t_{1}<\cdots<t_{\ell}, x_{1}<\cdots<x_{\ell}
$$

For a given bounded measurable control function $\psi:[0,1] \times \mathbb{R} \rightarrow \mathbb{R}_{+}$, an online strategy selecting such increasing sequence is defined by the following intuitive rule. Let $x$ be the last mark selected before time $t$, or some given $x_{0}$ if no selection has been made. Given the next mark $x^{\prime}$ is observed at time $t$, this mark is selected if and only if

$$
x<x^{\prime} \leq x+\psi(t, x) .
$$

One can think of more general online strategies, with the acceptance window shaped differently from an interval or possibly depending on the history in a more complex way. Yet the considered class is sufficient for the sake of optimisation and can be further reduced to controls of a special type.

For a given control $\psi$, define $X(t)$ and $L(t)$ to be, respectively, the last mark selected and the number of marks selected within the time interval $[0, t]$. The process 
$X=(X(t), t \in[0,1])$, which we call the running maximum, is a time-inhomogeneous Markov process, jumping from the generic state $x$ at rate $\psi(t, x)$ to another state uniformly distributed on $[x, x+\psi(t, x)]$. The length process $L=(L(t), t \in[0,1])$ just counts the jumps of $X$; hence the bivariate process $(X, L)$ is also Markovian. Moreover, the conditional distribution of $((X(t), L(t)), t \geq s)$ depends on the pre- $s$ history only through $X(s)$.

Intuitively, the bigger $\psi$, the faster $X$ and $L$ increase. To enable comparisons of selection processes with different controls it is very convenient to couple them by means of an additive representation through another Poisson random measure $\Pi^{*}$, thought of as a reserve of positive increments. The underlying properties of the planar Poisson process are translation invariance and spatial independence: $\Pi$ restricted to the shifted quadrant $(t, x)+\mathbb{R}_{+}^{2}$ is independent of $\Pi_{\mid[0, t] \times \mathbb{R}}$ and has the same distribution as the translation of $\Pi_{\mid \mathbb{R}_{+}^{2}}$ by vector $(t, x)$. So, letting $\Pi^{*}$ to be a distributional copy of $\Pi$, a solution to the system of stochastic differential equations

$$
\mathrm{d} X(t)=\int_{0}^{\psi(t, X(t))} x \Pi^{*}(\mathrm{~d} t \mathrm{~d} x), \quad \mathrm{d} L(t)=\int_{0}^{\psi(t, X(t))} \Pi^{*}(\mathrm{~d} t \mathrm{~d} x)
$$

with initial values $X(0)=x_{0}$ and $L(0)=0$ will have the same distribution as $(X, L)$.

By virtue of the additive realisation through $\Pi^{*}$, the online increasing subsequence problem is transformed into an online knapsack packing problem [10]. Here, the generic item of some size $x$ observed at time $t$ (an atom of $\Pi^{*}$ ) can be either packed or dismissed. The objective translates as maximisation of the expected number of items added within the unit time horizon to a knapsack of unit capacity. Note that for the increasing subsequence problem the (continuous) distribution of marks does not matter, while the knapsack problem is not distribution-free.

Lemma 1. For $i=1,2$ let $X_{i}$ be selection processes driven by controls $\psi_{i}$. By coupling via (4), each time a process with smaller acceptance window jumps, the other process also has a jump of the same size.

Proof. Straight from (4),

$$
\mathrm{d}\left(X_{1}-X_{2}\right)=\operatorname{sgn}\left(\psi_{1}-\psi_{2}\right) \int_{\psi_{1} \wedge \psi_{2}}^{\psi_{1} \vee \psi_{2}} x \Pi^{*}(\mathrm{~d} t \mathrm{~d} x)
$$

where for shorthand $\psi_{i}=\psi_{i}\left(t, X_{i}(t)\right)$.

Conditionally on $(X(s), L(s))=(x, \ell)$, the process $(X(s+\cdot)-x, L(s+\cdot)-\ell))$ has the same distribution as $\left(X^{(s, x)}, L^{(s, x)}\right)$, which similarly to (4) is given by

$$
d X^{(s, x)}(u)=\int_{0}^{\psi\left(s+u, x+X^{(s, x)}(u)\right)} y \Pi^{*}(\mathrm{~d} u \mathrm{~d} y), \quad \mathrm{d} L^{(s, x)}(u)=\int_{0}^{\psi\left(s+u, x+X^{(s, x)}(u)\right)} \Pi^{*}(\mathrm{~d} u \mathrm{~d} y) .
$$

Averaging, we obtain formulas for the predictable compensators of $X$ and $L$

$$
C_{X}(t):=\frac{\nu}{2} \int_{0}^{t} \psi^{2}(s, X(s)) \mathrm{d} s, \quad C_{L}(t):=\nu \int_{0}^{t} \psi(s, X(s)) \mathrm{d} s,
$$


so $X-C_{X}, L-C_{L}$ are zero-mean martingales.

With every control we may further relate a zero-mean martingale

$$
M(t):=L(t)+\mathbb{E}\{L(1)-L(t) \mid X(t)\}-\mathbb{E} L(1)
$$

with terminal value $L(1)-\mathbb{E} L(1)$. If $\psi$ does not depend on $x, L$ has independent increments and $M(t)=L(t)-\mathbb{E} L(t)$.

The selected increasing chain fits in the unit square if $X(1) \leq 1$, which translates in terms of the control function as the condition of feasibility:

$$
0<\psi(t, x) \leq 1-x \text { for }(t, x) \in[0,1]^{2}
$$

In the sequel, if not stated otherwise we set $x_{0}=0$ and only consider feasible controls.

\subsection{Principal convergence of the moments}

This section follows closely the arguments found in [8], pp. 291-292.

Let

$$
p(t):=\mathbb{E} X(t)=\mathbb{E} C_{X}(t), \quad q(t):=\frac{\mathbb{E} L(t)}{\sqrt{2 \nu}}=\frac{\mathbb{E} C_{L}(t)}{\sqrt{2 \nu}} .
$$

Some general relations between the moments follow straight from formulas for the compensators (5). For shorthand, write $\psi=\psi(X(s), s)$. We have

$$
0 \leq \mathbb{E} \int_{0}^{t}(1 \pm \sqrt{\nu / 2} \psi)^{2} \mathrm{~d} s=t \pm 2 q(t)+p(t)
$$

where the right-hand side in increasing in $t$. It follows,

$$
p(t)-t \geq 2(q(t)-t)
$$

Using the Cauchy-Schwarz inequality

$$
\begin{array}{r}
(p(t)-t)^{2}=\left(\mathbb{E} \int_{0}^{t}\left(1-\frac{\nu}{2} \psi^{2}\right) \mathrm{d} s\right)^{2} \leq \\
\mathbb{E} \int_{0}^{t}(1+\sqrt{\nu / 2} \psi)^{2} \mathrm{~d} s \mathbb{E} \int_{0}^{t}(1-\sqrt{\nu / 2} \psi)^{2} \mathrm{~d} s= \\
(t+2 q(t)+p(t))(t-2 q(t)+p(t)) .
\end{array}
$$

Similarly

$$
(q(t)-t)^{2}=\left(\mathbb{E} \int_{0}^{t} 1 \cdot(1-\sqrt{\nu / 2} \psi) \mathrm{d} s\right)^{2} \leq t(t-2 q(t)+p(t))
$$

The above relations did not use the feasibility constraint. For feasible control we have $p(1)<1$, hence from (7) also $q(1)<1$. Since all factors in the right-hand sides of (8), (9) are increasing, replacing them by their maximal values at $t=1$ we obtain

$$
(p(t)-t)^{2}<8(1-q(1)), \quad(q(t)-t)^{2}<2(1-q(1)) .
$$


We say that a strategy $\psi=\psi_{\nu}$ is asymptotically optimal in the principal term if $q(1) \rightarrow 1$, as $\nu \rightarrow \infty$, i.e. $\mathbb{E} L_{\nu}(1) \sim \sqrt{2 \nu}$; in that case 10 imply the uniform convergence of the moments

$$
\sup _{t \in[0,1]}|p(t)-t| \rightarrow 0, \sup _{t \in[0,1]}|q(t)-t| \rightarrow 0
$$

It follows from (1) that under the optimal strategy

$$
1-q(1) \sim \frac{\log \nu}{12 \sqrt{2 \nu}}, \quad \nu \rightarrow \infty
$$

This relation can be called a two-term asymptotic optimality. Whenever this holds, the general bounds 10 imply that both $\sup _{t \in[0,1]}|p(t)-t|$ and $\sup _{t \in[0,1]}|q(t)-t|$ can be estimated as $O\left(\sqrt{\log \nu} / \nu^{1 / 4}\right)$. A refinement of the convergence rate will be obtained in Section 7.

\subsection{The greedy strategy}

The greedy strategy, with control $\psi(t, x)=1-x$, outputs the sequence of consecutive records. The strategy is optimal for $\nu<1.34 \ldots$ Statistical properties of records from the Poisson process is a much-studied subject [9]. It is well known that, as $\nu$ increases, the distribution of $L(1)$ approaches normal with mean and variance both asymptotic to $\log \nu$. Normalisation (3) is not appropriate here as most of the records concentrate near the north-west corner of the unit square.

\subsection{The stationary strategy}

We call the strategy with control $\psi(t, x)=\sqrt{2 / \nu}$ stationary. Although not feasible, the stationary strategy is an important benchmark. Clearly, $L$ is a Poisson counting process with intensity $\mathbb{E} L(1)=\sqrt{2 \nu}$. Taking general constant control $\psi(t, x)=\sqrt{c / \nu}$ with some $c>0$ will yield a strategy outputting the mean length $\sqrt{\{c \wedge(2 / c)\} \nu}$, which is maximal for $c=2$. In fact, a much stronger optimality property holds: the stationary strategy achieves the maximum expected length over the class of strategies that satisfy the meanvalue constraint $\mathbb{E} X(1) \leq 1$, see [1, 4, 13, 14] for proof and generalisations. This gives the well-known upper bound mentioned in the Introduction, because each feasible strategy meets the mean-value constraint.

It is seen from (4) that $X$ is a compound Poisson process

$$
X(t)=\sqrt{\frac{2}{\nu}} \sum_{i=0}^{L(t)} U_{i}
$$

where $U_{1}, U_{2}, \ldots$ are independent of $L$, uniformly distributed on [0,1]. Straightforward calculation of moments using Wald's identities yields

$$
\mathbb{E} X(t)=t, \quad \operatorname{Var} X(t)=\frac{2^{3 / 2} t}{3 \sqrt{\nu}}, \quad \operatorname{Cov}(X(t), L(t))=t
$$


Since $(X, L)$ has independent increments, a functional limit in the Skorohod topology on $D[0,1]$ follows easily from the multidimensional invariance principle:

$$
(\widetilde{X}, \widetilde{L}) \Rightarrow\left(W_{1}, W_{2}\right), \text { as } \nu \rightarrow \infty
$$

where $\Rightarrow$ denotes weak convergence, and the limit process $\boldsymbol{W}:=\left(W_{1}, W_{2}\right)$ is a twodimensional Brownian motion with zero drift and covariance matrix

$$
\mathbb{E}\left\{\boldsymbol{W}(t)^{T} \boldsymbol{W}(t)\right\}=t \boldsymbol{\Sigma}, \text { where } \boldsymbol{\Sigma}:=\left(\begin{array}{cc}
\frac{2 \sqrt{2}}{3} & \frac{1}{\sqrt{2}} \\
\frac{1}{\sqrt{2}} & \frac{1}{\sqrt{2}}
\end{array}\right)
$$

So, marginally, $W_{1}$ and $W_{2}$ are centred Brownian motions with diffusion coefficients and correlation, respectively,

$$
\sigma_{1}:=\frac{2^{3 / 4}}{\sqrt{3}}, \quad \sigma_{2}:=\frac{1}{2^{1 / 4}}, \quad \rho:=\frac{\sqrt{3}}{2} .
$$

Notably,

$$
\rho=\frac{\sigma_{2}}{\sigma_{1}}
$$

which implies that the process $\boldsymbol{W}$ satisfies the identity

$$
2 W_{2}-W_{1} \stackrel{d}{=} W_{1}
$$

which has a pre-limit analogue

$$
2 \widetilde{L}-\tilde{X} \stackrel{d}{=} \widetilde{X}
$$

Identity 15 can be explained by the symmetry of the uniform distribution, $U_{i} \stackrel{d}{=} 1-U_{i}$, which allows us to write

$$
X(t) \stackrel{d}{=} \sqrt{\frac{2}{\nu}} \sum_{i=0}^{L(t)}\left(1-U_{i}\right)=\sqrt{\frac{2}{\nu}} L(t)-X(t) .
$$

Martingale (6) just coincides with naturally centred $L$.

The correlated Brownian motion has appeared in Bruss and Delbaen [8] (Theorem $4.1)$, as the limit of $(X, L)$ centred by their compensators $C_{X}$ and $C_{L}$ under the optimal (feasible) strategy. This connection confirms that the key to the fluctuation problem is understanding the nature of the drift component.

\subsection{A feasible version of the stationary strategy}

The strategy driven by $\psi(t, x)=\sqrt{2 / \nu} \wedge(1-x)$ is a counterpart of that introduced by Samuels and Steele in the discrete-time setting [21]. This is a minor modification of the stationary strategy to meet the feasibility condition. Define the hitting time

$$
\tau:=\inf \{t \in[0,1]: X(t) \geq 1-\sqrt{2 / \nu}\}
$$


with the convention inf $\varnothing=1$. The strategy acts as the stationary before $\tau$, and if $\tau<1$ proceeds with a greedy selection, so, in essence, the selection process is frozen at time $\tau$. Using elementary renewal theory arguments, we find asymptotics of the moments

$$
\mathbb{E} L(1) \sim \sqrt{2 \nu}-\frac{2^{3 / 4}}{\sqrt{3 \pi}} \nu^{1 / 4}, \quad \operatorname{Var} L(1) \sim \frac{2^{3 / 2}}{3}\left(1-\frac{1}{\pi}\right) \sqrt{\nu} .
$$

Hence the strategy is asymptotically optimal in the principal term.

Furthermore, $\widetilde{L}(1)$ converges in distribution to $2^{-1 / 4}\left\{\left(\xi_{1} / \sqrt{3}\right) \wedge \xi_{2}\right\}$, where $\xi_{1}, \xi_{2}$ are independent $\mathcal{N}(0,1)$, see [14]. The normalised terminal value $\widetilde{X}(1)$ is nonpositive, and converges in distribution to $-2^{3 / 4} 3^{-1 / 2}(\eta)_{+}$, where $\eta$ is another standard normal variable and $(\cdot)_{+}$denotes the positive part. By symmetry of the normal distribution, the hitting time $\tau$ assumes value 1 with probability approaching $\mathbb{P}\left(V_{1}(1)<0\right)=1 / 2$, and otherwise $1-\tau$ is of the order $\nu^{-1 / 4}$. Comparing with the stationary strategy, one can see that there is an optimality gap of order $\nu^{1 / 4}$ which occurs due to a premature freeze of selection in the event $\tau<1$.

Note that the moments of terminal values satisfy $1-p(1) \sim c_{1} \nu^{-1 / 4}, 1-q(1) \sim c_{2} \nu^{-1 / 4}$ with some $c_{1}, c_{2}>0$, while 10 overestimates the first as $1-p(1)=O\left(\nu^{-1 / 8}\right)$.

In terms of the normalised running maximum, $\tau$ is the time when $\widetilde{X}$ hits the straight line connecting points $\left(0, \nu^{1 / 4}\right)$ and $(1,0)$. Since $\tau \rightarrow 1$ in probability, $(\widetilde{X}, \widetilde{L})$ has the same functional limit as under the stationary strategy on every interval $[0,1-h]$, for $h \in(0,1)$. Extending the functional limit to the closed $[0,1]$ leads to a discontinuity at $t=1$. To capture the jump, it is enough to modify the correlated Brownian motion $\boldsymbol{W}$ by replacing the terminal value $\left(W_{1}(1), W_{2}(1)\right)$ with

$$
\left(W_{1}(1)-\left(W_{1}(1)\right)_{+}, W_{2}(1)-(2 / \sqrt{3})\left(W_{1}\right)_{+}\right)
$$

\subsection{Self-similar asymptotically optimal strategies}

We call strategy self-similar if the control $\psi=\psi_{\nu}$ has the form

$$
\psi(t, x):=(1-x) \delta(\nu(1-t)(1-x)), \quad(t, x) \in[0,1]^{2} .
$$

for some function $\delta: \mathbb{R}_{+} \rightarrow[0,1]$. Note that such a strategy is feasible and $\psi_{\nu}(0,0)=\delta(\nu)$. The rationale behind this definition is the following. Assuming $x$ to be the running maximum at time $t$, the remaining part of the chain should be selected from the northeast rectangle spanned on $(t, x)$ and $(1,1)$, and by the optimality principle the subsequence selected from the rectangle should have maximal expected length. Mapping the rectangle onto $[0,1]^{2}$ it is readily seen that the subproblem is an independent replica of the original problem of optimal selection from the unit square with intensity parameter $\nu(1-x)(1-t)$. The martingale (6) assumes the form

$$
M(t)=L(t)+F(\nu(1-t)(1-X(t))-F(\nu),
$$

where the value function $F$, for given control, depends on one variable

$$
F(\nu):=\mathbb{E} L_{\nu}(1), \quad F(0)=0 .
$$


Assumption. From this point on we assume that the selection strategy is self-similar as defined by (16), with function $\delta$ having asymptotics

$$
\delta(\nu)=\sqrt{2 / \nu}+O\left(\nu^{-1}\right), \quad \nu \rightarrow \infty .
$$

The assumption is central and deserves comments. Whenever $\nu(1-x)(1-t)$ is large, (18) implies asymptotics of the control

$$
\psi(t, x) \sim \sqrt{\frac{2(1-x)}{\nu(1-t)}},
$$

which shows that near the diagonal $x=t$ the acceptance window is about the same as for the stationary strategy. Away from the diagonal, the acceptance window is close to that for the stationary strategy adjusted to the rectangle north-east of $(t, x)$.

It is known [14] that the optimal strategy satisfies the asymptotic expansion

$$
\delta^{*}(\nu) \sim \sqrt{2 / \nu}-(3 \nu)^{-1}+O\left(\nu^{-3 / 2}\right) .
$$

A minor adjustment of Theorem 6 in [14] shows that if we assume, more generally, the relation $\delta(\nu) \sim \sqrt{2 / \nu}+\beta / \nu$ with some parameter $\beta \in \mathbb{R}$, then asymptotic expansions of the moments (1), (2) are still valid, with only constant terms depending on $\beta$. Using a sandwich argument based on Lemma 1, one can further show that under the assumption (18) expansions of the moments hold but with constant terms being replaced by some $O(1)$ remainders. In particular, condition (18) ensures the two-term asymptotic optimality (11), equivalent to the asymptotic expansion of the value function,

$$
F(\nu)=\sqrt{2 \nu}-\frac{1}{12} \log (\nu+1)+O(1)
$$

We stress that the logarithmic term here (as well as in the counterpart of the variance formula (2) ) is not affected by the remainder in (18), rather appears due to the self-similar adjustment of (a feasible version of) the stationary strategy, as incorporated in (19). The impact of the second term in (18) on moments of the running maximum will be scrutinised in Section 7.

Approximation 19 is not useful when $t$ or $x$ are too close to 1 , so that $\nu(1-t)(1-x)$ varies within $O(1)$. To embrace the full range of the variables, for the sequel we choose $\beta>1$ large enough to meet the bounds

$$
\left|\psi(t, x)-\sqrt{\frac{2(1-x)}{\nu(1-t)}}\right|<\frac{\beta}{\nu(1-t)}, \quad \text { for }(t, x) \in[0,1) \times[0,1) .
$$

This will be employed along with the bound

$$
\psi(t, x)<\frac{1}{\nu(1-t)}, \text { for } 1-x<\frac{1}{\nu(1-t)}
$$

which follows by feasibility. 


\section{Generators}

The selection process in Section 2.4 demonstrates one type of possible pathology, caused by large overshooting the diagonal at times close to $t=1$. But under (18) it is not even

obvious that $(\tilde{X}, \widetilde{L})$ has a sensible limit in $D[0,1]$. A major technical difficulty in showing the convergence is the singularity of $(19)$ at $t=1$. This will be handled in two steps. First, we bound the time variable away from $t=1$ and show the convergence of the generators on a sufficiently big space of test functions. Then we will apply domination arguments to bound fluctuations near the right endpoint, thus justifying convergence on the full $[0,1]$.

The processes we consider are not time-homogeneous, therefore by computing generators we include the time variable in the state vector. From (4), the generator of the jump process $(X, L)$ is

$$
\mathcal{L}_{\nu} f(t, x, \ell)=f_{t}(t, x, \ell)+\nu \int_{0}^{\psi(t, x)}\{f(t, x+u, \ell+1)-f(t, x, \ell)\} \mathrm{d} u .
$$

For the processes centered by $t$ we should include $-f_{x}-f_{\ell}$ in the generator. Then, with the change of variables

$$
x \rightarrow x \nu^{-1 / 4}+t, \ell \rightarrow\left(\ell \nu^{-1 / 4}+t\right) \sqrt{2 \nu}, \quad \widetilde{\psi}(t, x):=\nu^{1 / 4} \psi\left(t, x \nu^{-1 / 4}+t\right)
$$

we arrive at the generator of $(\widetilde{X}, \widetilde{L})$

$$
\widetilde{\mathcal{L}}_{\nu} f=f_{t}-\nu^{-1 / 4}\left(f_{x}+f_{\ell}\right)+\nu^{3 / 4} \int_{0}^{\widetilde{\psi}(t, x)}\{f(t, x+u, \ell+v)-f(t, x, \ell)\} \mathrm{d} u,
$$

where we abbreviate $f=f(t, x, \ell)$ etc., and

$$
v:=(4 \nu)^{-1 / 4}
$$

We extend $\widetilde{\mathcal{L}}_{\nu} f$ by 0 outside the reachable range of $(\widetilde{X}, \widetilde{L})$. Note that the range of $\widetilde{X}(t)$ lies within the bounds

$$
-t \nu^{1 / 4} \leq x \leq(1-t) \nu^{1 / 4}
$$

We fix $h \in(0,1)$ and focus on $t \in[0,1-h]$, so achieving uniformly in this range

$$
\widetilde{\psi}(t, x)=O\left(\nu^{-1 / 4}\right)
$$

and for $k \geq 1$

$$
\widetilde{\psi}^{k}(t, x)=\left(2-\frac{2 x}{\nu^{1 / 4}(1-t)}\right)^{k / 2} \nu^{-k / 4}+O\left(\nu^{-(k+2) / 4}\right), \text { for } x \leq(1-t) \nu^{1 / 4}-\frac{1}{\nu^{3 / 4}(1-t)}
$$

as dictated by the bounds $(22),(23)$.

Now let $\mathcal{D}$ be the space of vanishing at infinity functions $f \in C_{0}^{3}\left([0,1] \times \mathbb{R}^{2}\right)$ which satisfy a rapid decrease property

$$
\sup \left|x^{k} f_{\bullet}(t, x, \ell)\right|<\infty,
$$


where $f_{\bullet}$ is any derivative of $f$ of the first or second order and $k>0$. Set $D_{h, \nu}^{>}:=\left\{(t, x, \ell): t \in[0,1-h],|x|>\nu^{1 / 16}\right\}, \quad D_{h, \nu}^{<}:=\left\{(t, x, \ell): t \in[0,1-h],|x| \leq \nu^{1 / 16}\right\}$.

We shall be using that for $f \in \mathcal{D}$

$$
\lim _{\nu \rightarrow \infty} \sup _{D_{h, \nu}^{>}}\left|\nu^{k} f_{\bullet}(x)\right|=0
$$

The integrand in 24) expands as

$$
f(t, x+u, \ell+v)-f(t, x, \ell)=f_{x} u+f_{\ell} v+\frac{1}{2} f_{x x} u^{2}+f_{x \ell} u v+\frac{1}{2} f_{\ell \ell} v^{2}+R,
$$

where the remainder can be estimated as

$$
|R| \leq c \sum_{i=0}^{3} u^{i} v^{3-i}
$$

with constant $c$ chosen bigger than the maximum absolute value of any third derivative of $f$. Hence for the integrated remainder we have a uniform estimate

$$
\nu^{3 / 4}\left|\int_{0}^{\widetilde{\psi}} R d u\right| \leq \nu^{3 / 4} c \sum_{i=1}^{4} \widetilde{\psi}^{i} v^{4-i}=O\left(\nu^{-1 / 4}\right),
$$

using (26), (25).

Integrating the Taylor polynomial yields

$\widetilde{\mathcal{L}}_{\nu} f=f_{t}-\nu^{1 / 4}\left(f_{x}+f_{\ell}\right)+\nu^{3 / 4}\left\{\frac{1}{2} f_{x} \widetilde{\psi}^{2}+f_{\ell} v \widetilde{\psi}+\frac{1}{6} f_{x x} \widetilde{\psi}^{3}+\frac{1}{2} f_{x \ell} \widetilde{\psi}^{2} v+\frac{1}{2} f_{\ell \ell} v^{2} \widetilde{\psi}\right\}+O\left(\nu^{-1 / 4}\right)$.

Applying 28

$$
\lim _{\nu \rightarrow \infty} \sup _{D_{h, \nu}^{>}}\left|\widetilde{\mathcal{L}}_{\nu} f(t, x, \ell)\right|=0 .
$$

Thus we focus on the range $D_{h, \nu}^{<}$, where $(22)$ and $(27)$ can be employed. From $(22)$

$$
-\nu^{-1 / 4} f_{x}+\nu^{3 / 4} \frac{1}{2} f_{x} \widetilde{\psi}^{2}=-\frac{x}{1-t} f_{x}+O\left(\nu^{-1 / 4}\right)
$$

Observing that in this range $\left|x \nu^{-1 / 4}\right| \leq \nu^{-3 / 16}$ for $k>0$ we expand as

$$
\widetilde{\psi}^{k}(t, x, \ell)=2^{k / 2} \nu^{-k / 4}-\frac{2^{k / 2-1} x}{1-t} \nu^{-(k+1) / 4}+O\left(\nu^{-(k+1) / 4-1 / 8}\right),
$$

with the remainder estimate being uniform over $D_{h, \nu}^{<}$. The remaining calculations is a careful book-keeping using this formula and that the derivatives are uniformly bounded: 


$$
\begin{aligned}
-\nu^{1 / 4} f_{\ell}+\nu^{3 / 4} f_{\ell} v \widetilde{\psi} & =-\frac{x}{2(1-t)} f_{\ell}+O\left(\nu^{-1 / 8}\right), \\
\nu^{3 / 4} \frac{1}{6} f_{x x} \widetilde{\psi}^{3} & =\frac{\sqrt{2}}{3} f_{x x}+O\left(\nu^{-3 / 16}\right), \\
\nu^{3 / 4} \frac{1}{2} f_{x \ell} \widetilde{\psi}^{2} v & =\frac{1}{\sqrt{2}} f_{x \ell}+O\left(\nu^{-3 / 16}\right), \\
\nu^{3 / 4} \frac{1}{2} f_{\ell \ell} v^{2} \widetilde{\psi} & =\frac{1}{2 \sqrt{2}} f_{\ell \ell}+O\left(\nu^{-3 / 16}\right) .
\end{aligned}
$$

Define operator

$$
\widetilde{\mathcal{L}} f:=f_{t}-\frac{x}{1-t} f_{x}-\frac{x}{2(1-t)} f_{\ell}+\frac{\sigma_{1}^{2}}{2} f_{x x}+\frac{\sigma_{2}^{2}}{2} f_{\ell \ell}+\sigma_{1} \sigma_{2} \rho f_{x \ell},
$$

with $\sigma_{1}, \sigma_{2}$, and $\rho$ given by 13 .

Lemma 2. For $f \in \mathcal{D}$ and $h \in(0,1)$

$$
\lim _{\nu \rightarrow \infty} \sup _{(t, x, \ell) \in[0,1-h] \times \mathbb{R}^{2}}\left|\widetilde{\mathcal{L}}_{\nu} f(t, x, \ell)-\widetilde{\mathcal{L}} f(t, x, \ell)\right|=0
$$

Proof. The supremum over $D_{h, \nu}^{>}$goes to zero since by (28) the analogue of $(29)$ holds true for $\widetilde{\mathcal{L}}$. The supremum over $D_{h, \nu}^{<}$goes to zero by the above expansions.

Operator $\widetilde{\mathcal{L}}$ is the generator of a Gaussian diffusion process which satisfies the stochastic differential equation

$$
\begin{aligned}
\mathrm{d} Y_{1}(t) & =-\frac{Y_{1}(t)}{1-t} \mathrm{~d} t+\mathrm{d} W_{1}(t) \\
\mathrm{d} Y_{2}(t) & =-\frac{Y_{1}(t)}{2(1-t)} \mathrm{d} t+\mathrm{d} W_{2}(t)
\end{aligned}
$$

with zero initial value, where $\boldsymbol{W}=\left(W_{1}, W_{2}\right)$ is the two-dimensional Brownian motion with covariance $\boldsymbol{\Sigma}$ introduced in $(12)$.

From the equation for the first component (30), it is seen that $Y_{1}$ is a Brownian bridge

$$
Y_{1}(t)=(1-t) \int_{0}^{t} \frac{\mathrm{d} W_{1}(s)}{1-s}
$$

with the covariance function $\operatorname{Cov}\left(Y_{1}(s), Y_{1}(t)\right)=\sigma_{1} s(1-t), 0 \leq s \leq t \leq 1$. In particular, $Y_{1}(1)=0$. We shall discuss the second component later on.

The space $\mathcal{D}$ is dense in a larger space $C_{0}^{3}\left([0,1-h] \times \mathbb{R}^{2}\right)$. Since the differentiability properties of functions are preserved under averaging over normally distributed translations, $\mathcal{D}$ is invariant under the semigroup of $\boldsymbol{Y}$. Thus by Watanabe's theorem (see [18], 
Proposition 17.9) $\mathcal{D}$ is a core of operator $\widetilde{\mathcal{L}}$. The above Lemma 2 and Theorem 17.25 from [18] now imply weak convergence

$$
\left(\widetilde{X}_{\nu}, \widetilde{L}_{\nu}\right) \Rightarrow\left(Y_{1}, Y_{2}\right) \text { in } D[0,1-h]
$$

for every $h \in(0,1)$. A closer inspection of the above approximation errors suggests that the quality of convergence deteriorates as $h \rightarrow 0$.

We encountered the Brownian motion $\boldsymbol{W}$ in connection with the free-endpoint stationary strategy in Sections 2.3 and 2.4. Now we see that the variable control (19) causes a drift that, in the $\nu \rightarrow \infty$ limit, forces the running maximum to timely arrive at the north-east corner of the square.

\section{Convergence to diffusion: end of the proof}

The martingale problem for $\widetilde{\mathcal{L}}$ is well-posed on the complete interval, and the SDE 30 has a unique strong solution. This suggests to extend convergence (33) to the full $[0,1]$. To that end, we need to monitor the behaviour of $\widetilde{\mathcal{L}}_{\nu} f$ for $t$ close to 1 . Estimates in Bruss and Delbaen ([8], p. 294) show that $\widetilde{X}_{\nu}(1) \rightarrow 0$ in probability, which agrees neatly with the Brownian bridge limit, but this still does not exclude giant fluctuations of the pre-limit process near $t=1$.

A similar kind of difficulty appears by the martingale approach to the classic problem of convergence of the empirical distribution function [15, 16]. The proof found in Jacod and Shiryaev (see [16], p.561) handles the nuisance by exploiting the time reversibility of the Brownian bridge. Our argument will rely on the self-similarity.

Since 33 entails convergence of finite-dimensional distributions for times $t<1$ and ensures that the modulus of continuity behaves properly over $[0,1-h]$, to justify tightness of $\widetilde{X}_{\nu}$ 's, and hence their convergence on $[0,1]$, it will be enough to show that

$$
\lim _{h \rightarrow 0} \limsup _{\nu} \mathbb{P}\left(\sup _{t \in[1-h, 1]}\left|\widetilde{X}_{\nu}(t)\right|>h^{1 / 4}\right)=0 .
$$

Define $\xi_{\nu, h}$ by setting

$$
\tilde{X}_{\nu}(1-h)=\sigma_{1} \sqrt{h(1-h)} \xi_{\nu, h} .
$$

Since $\widetilde{X}_{\nu}(1-h) \stackrel{d}{\rightarrow} Y_{1}(1-h)$ the distribution of $\xi_{\nu, h}$ is close to $\mathcal{N}(0,1)$ for large $\nu$.

By self-similarity of the selection strategy, $\left(\left(X_{\nu}(t)-t\right), t \in[1-h, 1]\right)$ has the same distribution as $\left(h^{-1}\left(X_{\nu h^{2}}(t)-t\right), t \in[0,1]\right)$ with the initial value $X_{\nu h^{2}}(0)=$ $\nu^{-1 / 4} \sigma_{1} \sqrt{(1-h) / h} \xi_{\nu, h}$, as is seen by zooming in the corner square north-east of the point $(1-h, 1-h)$ with factor $h^{-1}$. Changing variable $\nu h^{2} \rightarrow \nu, 34$ translates as a compact containment condition

$$
\lim _{h \rightarrow 0} \limsup _{\nu} \mathbb{P}\left(\sup _{t \in[0,1]}\left|\widetilde{X}_{\nu}(t)\right|>h^{-1 / 4}\right)=0
$$

under the initial value $\widetilde{X}_{\nu}(0)=\sqrt{1-h} \xi_{\nu, h}$.

To verify 35 we shall squeeze the running maximum $X$ between $X^{\downarrow}$ and $X^{\uparrow}$ whose normalised versions satisfy the compact containment condition. We force the majorant 
and the minorant to live on the opposite sides of the diagonal. Both have independent, almost stationary increments, so that functional limits can be readily identified. For simplicity we will assume $X_{\nu}(0)=0$. The general case with $X_{\nu}(0)$ of the order $\nu^{-1 / 4}$ can be handled by the same method.

\subsection{Majorant}

Define process $X^{\uparrow}=X_{\nu}^{\uparrow}$ as solution to

$$
\mathrm{d} X^{\uparrow}(t)=\int_{0}^{\psi^{\uparrow}(t)} x \Pi^{*}(\mathrm{~d} t \mathrm{~d} x)+1\left(X^{\uparrow}(t)=t\right) \mathrm{d} t,
$$

$X^{\uparrow}(0)=K \nu^{-1 / 2}$ for some big enough $K>0$, with control

$$
\psi^{\uparrow}(t):=\sqrt{\frac{2}{\nu}}+\frac{\beta}{\nu(1-t)} 1\left(t \leq 1-K \nu^{-1 / 2}\right)
$$

not depending on $x$. Notation $1(\cdots)$ is used for indicators. The process never drops below the line $x=K \nu^{-1 / 2}+t$, and whenever the line is hit the path drifts along it for some time. By the construction, above the diagonal the process $X^{\uparrow}$ increases faster than $X$, and is, in fact, a majorant.

Lemma 3. By coupling via (4), $X^{\uparrow} \geq X$ a.s.

Proof. By the virtue of (22), (23) and definition of $\psi^{\uparrow}$ we have $\psi^{\uparrow}(t, x)>\psi(t, x)$ for $x>t, t \leq 1-K \nu^{-1 / 2}$. Hence by Lemma 1 , $\mathrm{d}\left\{X^{\uparrow}(t)-X(t)\right\}>0$ conditional on $X^{\uparrow}(t)>X(t)>t$ at time $t<1-K \nu^{-1 / 2}$.

Initially $X^{\uparrow}(0)>X(0)$, and $X^{\uparrow}(t)>1>X(t)$ for $t>1-K \nu^{-1 / 2}$. Hence the only way the paths can cross is that $X$ overjumps $X^{\uparrow}$ from some position $x<t \leq X^{\uparrow}(t)$ at some time $t \leq 1-K \nu^{-1 / 2}$. The latter possibility is excluded, because

$$
\begin{aligned}
\psi(t, x) & <\sqrt{\frac{2}{\nu}}\left(1+\frac{t-x}{1-t}\right)+\frac{\beta}{\nu(1-t)}<\sqrt{\frac{2}{\nu}}\left(1+\frac{t-x}{2(1-t)}\right)+\frac{\beta}{\nu(1-t)} \\
& \leq \sqrt{\frac{2}{\nu}}+\frac{t-x}{K \sqrt{2}}+\frac{\beta}{K \sqrt{\nu}}<t-x+\frac{K}{\sqrt{\nu}}
\end{aligned}
$$

for $K$ chosen big enough.

Let

$$
S(t):=\int_{0}^{t} \int_{0}^{\psi^{\uparrow}(t)} x \Pi^{*}(\mathrm{~d} s \mathrm{~d} x)-t .
$$

This is a process with independent increments, which we can split into two independent components

$$
S(t)=\left(\int_{0}^{t} \int_{0}^{\sqrt{2 / \nu}} x \Pi^{*}(\mathrm{~d} s \mathrm{~d} x)-t\right)+\int_{0}^{t} \int_{\sqrt{2 / \nu}}^{\psi^{\uparrow}(t)} x \Pi^{*}(\mathrm{~d} s \mathrm{~d} x)
$$


The mean value of the second part is estimated as

$$
\frac{2 \nu}{\sqrt{\nu}} \int_{0}^{1-K / \sqrt{\nu}} \frac{\beta}{\nu(1-t)} \mathrm{d} t=O\left(\frac{\log \nu}{\sqrt{\nu}}\right)
$$

and the first is a compensated compound Poisson process. Thus $\nu^{1 / 4} S \Rightarrow W_{1}$ as $\nu \rightarrow \infty$.

Processes akin to $\left(X^{\uparrow}(t)-t, t \in[0,1]\right)$ are common in applied probability [3, 5]. In particular, by the interpretation as the content of a single-server $M / G / 1$ queue, the positive increments present jobs that arrive by Poisson process and are measured in terms of the demand on the service time. The downward drift occurs due to the unit processing rate when the server is busy. Borrowing a useful identity,

$$
X^{\uparrow}(t)-t=S(t)-\inf _{u \in[0, t]} S(u)
$$

we conclude on the weak convergence $\left(\nu^{1 / 4}\left(X^{\uparrow}(t)-t\right), t \in[0,1]\right) \Rightarrow\left|W_{1}\right|$ to a reflected Brownian motion.

\subsection{Minorant}

This time we define $X^{\downarrow}$ by 4 with control

$$
\psi^{\downarrow}(t, x)=\left\{\begin{array}{c}
\left(\sqrt{\frac{2}{\nu}}-\frac{\beta}{\nu(1-t)}\right) \wedge(t-x), \text { for } 0 \leq t \leq 1-K / \sqrt{\nu} \\
0, \text { for } 1-K / \sqrt{\nu}<t \leq 1 .
\end{array}\right.
$$

where $K$ is sufficiently large. We can regard this as a suboptimal strategy that never selects marks $x>t$. Starting at state 0 , the running maximum process stays below the diagonal throughout, and gets frozen at $t=1-K / \sqrt{\nu}$. A counterpart of Lemma 3 , $X^{\downarrow}<X$ a.s., is readily checked.

Switching general $\beta>0$ to $\beta=0$ impacts $\mathbb{E} X^{\downarrow}(t)$ by $O\left(\nu^{-1 / 2} \log \nu\right)$ uniformly in $t \in[0,1]$. Indeed, the jumps are bounded by $2 / \sqrt{\nu}$, and the expected number of jumps increases by $O(\log \nu)$.

Assuming $\beta=0$, the process $\left(X^{\downarrow}(t)-t, t \in\left[0,1-K \nu^{-1 / 2}\right]\right)$ is a compensated compound Poisson process on the negative halfline, with reflection at 0 . We have therefore

$$
\left(\nu^{1 / 4}\left(X^{\downarrow}(t)-t\right), t \in[0,1]\right) \Rightarrow-\left|W_{1}\right| \text {. }
$$

A rigorous proof can be obtained by inspecting convergence of the generator acting on the functions $f \in \mathcal{D}$ with $f_{x}(t, 0)=0$.

\subsection{The length process near termination}

Having established weak convergence of $\widetilde{X}$, we wish to estimate fluctuations of $\widetilde{L}$ near $t=1$. To that end, we aim to verify that

$$
\lim _{h \rightarrow 0} \limsup _{\nu} \mathbb{P}\left(\sup _{t \in[1-h, 1]}|\widetilde{L}(t)-\widetilde{L}(1-h)|>\epsilon\right)=0 .
$$


Write $s=1-h$ and split the difference in 36 in three parts

$$
\widetilde{L}(t)-\widetilde{L}(s)=\nu^{1 / 4} P_{1}(t)-\nu^{1 / 4} P_{2}(t)+\nu^{1 / 4} P_{3}(t),
$$

where

$$
\begin{aligned}
& P_{1}(t):=(2 \nu)^{-1 / 2}\{M(t)-M(s)\} \\
& P_{2}(t):=(2 \nu)^{-1 / 2} F(\nu(1-t)(1-X(t)))-(1-t) \\
& P_{3}(t):=(2 \nu)^{-1 / 2} F(\nu(1-s)(1-X(s)))-(1-s) .
\end{aligned}
$$

From 21,

$$
\lim _{\nu \rightarrow \infty} \sup _{z \in[0,1]} \nu^{1 / 4}\left|(2 \nu)^{-1} F(\nu z)-z\right|=0 .
$$

Using this, definition of $\tilde{X}$ and that $|1-\sqrt{1-z}| \leq|z|$ for $z<1$ we obtain

$$
\begin{aligned}
& \left|P_{2}(t)\right| \leq|\sqrt{(1-t)(1-X(t))}-(1-t)|+ \\
& \left\{(2 \nu)^{-1 / 2} F(\nu(1-t)(1-X(t)))-\sqrt{(1-t)(1-X(t))}\right\} \leq \\
& \mid(1-t)\left(\sqrt{\left.1-\frac{\tilde{X}(t)}{\nu^{1 / 4}(1-t)}-1\right)\left|+\sup _{z \in[0,1]}\right|(2 \nu)^{-1} F(\nu z)-z \mid \leq}\right. \\
& \nu^{-1 / 4}|\tilde{X}(t)|+\sup _{z \in[0,1]}\left|(2 \nu)^{-1} F(\nu z)-z\right|=\nu^{-1 / 4}|\tilde{X}(t)|+o\left(\nu^{-1 / 4}\right),
\end{aligned}
$$

so from 35

$$
\lim _{h \rightarrow 0} \limsup _{\nu} \mathbb{P}\left(\sup _{t \in[1-h, 1]} \nu^{1 / 4}\left|P_{2}(t)\right|>\varepsilon / 3\right)=0 .
$$

This relation also holds for $P_{3}$.

For the first part, apply Doob's maximal inequality

$$
\mathbb{P}\left(\sup _{t \in[1-h, 1]} \nu^{1 / 4}\left|P_{1}(t)\right|>\varepsilon / 3\right) \leq \frac{9}{2 \varepsilon^{2} \sqrt{\nu}} \operatorname{Var}\{M(1)-M(1-h)\} .
$$

In terms of the quadratic variation (see [6], Chapter 2)

$$
\operatorname{Var}\{M(1)-M(s)\}=\mathbb{E} \int_{s}^{1} \nu \psi(t, X(t)) \varphi(t, X(t)) \mathrm{d} t
$$

where

$$
\varphi(t, x)=\mathbb{E}\{1+F(\nu(1-t)(1-x-U \psi(t, x)))-F(\nu(1-t)(1-x))\}^{2}
$$

(with $U$ uniform on $[0,1]$ ) is the mean-square size of the generic jump of $M$. Under the optimal strategy $0 \leq \varphi(t, x) \leq 1$ (finer estimates are in [8], Section 4), and from (21) and (18) we have a uniform bound $|\varphi(t, x)|<c$. Whence

$$
\operatorname{Var}\{M(1)-M(1-h)\}<c \mathbb{E} \int_{1-h}^{1} \nu \psi(t, X(t)) \mathrm{d} t=c \mathbb{E}\{L(1)-L(1-h)\}<c \sqrt{2 \nu h},
$$

the probability in 38 is estimated as $O(\sqrt{h})$, and $(36$ follows from this and 37 . 


\section{Main result}

By the domination argument, tightness of $\left(\widetilde{X}_{\nu}, \widetilde{L}_{\nu}\right)$ follows on the whole [0,1], and we arrive at our main result.

Theorem 4. The normalised running maximum and the length process (3) driven by a control satisfying (16) and (18) (in particular, under the optimal online selection strategy) converge weakly in the Skorokhod space $D[0,1]$,

$$
\left(\widetilde{X}_{\nu}, \widetilde{L}_{\nu}\right) \Rightarrow\left(Y_{1}, Y_{2}\right), \quad \text { as } \quad \nu \rightarrow \infty
$$

where the limit bivariate process is a Gaussian diffusion defined by the equations (30), (31) with zero initial conditions.

We observed already that $Y_{1}$ is the Brownian bridge 32 and from 31

$$
Y_{2}(t)=\frac{Y_{1}(t)}{2}-\frac{W_{1}(t)}{2}+W_{2}(t)
$$

so splitting the martingale part in independent components, we get, explicitly,

$$
Y_{2}(t)=\int_{0}^{t} \frac{(1-t)}{2(1-s)} \mathrm{d} W_{1}(s)+\frac{1}{4} W_{1}(t)+\left(W_{2}(t)-\frac{3}{4} W_{1}(t)\right),
$$

which is a sum of a Brownian motion, derived Brownian bridge, and another independent Brownian motion.

To find the covariance structure, it is convenient to resort to matrix calculations. We may write the solution to 30 , (31) as

$$
\boldsymbol{Y}(t)^{T}=e^{a(t)} \int_{0}^{t} e^{-a(u)} \mathrm{d} \boldsymbol{W}(u)^{T}
$$

where

$$
a(t):=A \int_{0}^{t} \frac{1}{1-u} \mathrm{~d} u=A \log (1-t), \quad A:=\left(\begin{array}{cc}
1 & 0 \\
\frac{1}{2} & 0
\end{array}\right),
$$

which yields by the Itó isometry

$$
\mathbb{E}\left\{\boldsymbol{Y}(s)^{T} \boldsymbol{Y}(t)\right\}=\int_{0}^{t} e^{a(s)-a(u)} \boldsymbol{\Sigma} e^{(a(t)-a(u))^{T}} \mathrm{~d} u, \quad 0 \leq s \leq t \leq 1 .
$$

Since $A$ is an idempotent matrix, the exponents are readily calculated as

$$
\begin{aligned}
e^{a(t)} & =\sum_{i=0}^{\infty} \frac{A^{i}(\log (1-t))^{i}}{i !}=I+A \sum_{i=1}^{\infty} \frac{(\log (1-t))^{i}}{i !}=I-t A=\left(\begin{array}{cc}
1-t & 0 \\
-\frac{t}{2} & 1
\end{array}\right) \\
e^{-a(t)} & =1+\frac{t}{1-t} A=\left(\begin{array}{cc}
\frac{1}{1-t} & 0 \\
\frac{t}{2(1-t)} & 1
\end{array}\right) .
\end{aligned}
$$


With a minor help of Mathematica we arrive at the cross-covariance matrix

$$
\mathbb{E}\left\{\boldsymbol{Y}(s)^{T} \boldsymbol{Y}(t)\right\}=\left(\begin{array}{cc}
\frac{2 \sqrt{2} s(1-t)}{3} & \frac{2 s(1-t)-(1-s) \log (1-s)}{3 \sqrt{2}} \\
\frac{(1-t)(2 s-\log (1-s))}{3 \sqrt{2}} & \frac{2 s(2-t)-(2-s-t) \log (1-s))}{6 \sqrt{2}}
\end{array}\right)
$$

where $0 \leq s \leq t \leq 1$.

The following graphs illustrate the covariance structure of $\boldsymbol{Y}(t)$.

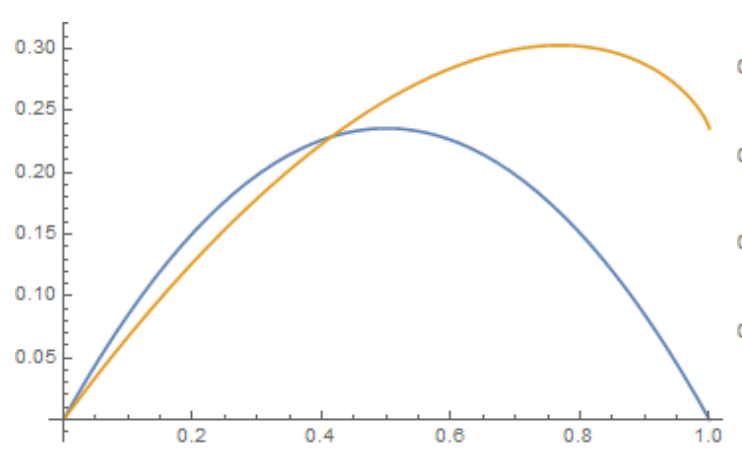

(a) $\operatorname{Var} Y_{1}(t), \operatorname{Var} Y_{2}(t)$



(b) $\operatorname{Cov}\left(Y_{1}(t), Y_{2}(t)\right), \operatorname{Corr}\left(Y_{1}(t), Y_{2}(t)\right)$

The limit length process $Y_{2}$ is not Markovian, since its covariance function does not satisfy the factorisation criterion (see [19], p. 148). The sum of two first terms in (39) is non-Markovian too.

\section{Derived processes}

From Theorem 4 follow functional limits for normalised compensators and martingale (6)

$$
\begin{aligned}
& \nu^{1 / 4}\left(C_{X}(t)-t, t \in[0,1]\right) \Rightarrow Y_{1}-W_{1}, \\
& 2 \nu^{1 / 4}\left(\frac{C_{L}(t)}{\sqrt{2 \nu}}-t, t \in[0,1]\right) \Rightarrow Y_{1}-W_{1} \text {, } \\
& \sqrt{2} \nu^{-1 / 4} M \Rightarrow 2 W_{2}-W_{1} \stackrel{d}{=} W_{1},
\end{aligned}
$$

with account of (14). A counterpart of $(15)$ becomes

$$
\widetilde{L}-2 \widetilde{X} \Rightarrow W_{1}
$$

Notably, the limit distributions for $t=1$ are all the same $\mathcal{N}\left(\sigma_{1}^{2}, 0\right)$.

For a normalised square-root process

$$
\widetilde{Z}(t):=\nu^{1 / 4}\left(\frac{Z(t)}{\sqrt{2 \nu}}-(1-t)\right)
$$

we have $\widetilde{Z} \Rightarrow-\frac{1}{2} Y_{1}$. In [14] we showed that the range of $Z$ at big distance from 0 can be split in almost independent renewal cycles with distribution close to that of $(E / 2+U) / \sqrt{2}$, where $E$ and $U$ are independent standard exponential and uniform variables. 
From these limit relations the result of [8] on the joint convergence of normalised compensated $X$ and $L$ to $\boldsymbol{W}$ easily follows. Bruss and Delbaen also proved the Brownian limit for the martingale $M$, which by virtue of $M(1)=L(1)-F(\nu)$ lead them to the central limit theorem for the total length $L(1)$.

It is of interest to look at the distributions of the pairs $\left(X(t), C_{X}(t)\right)$ and $\left(L(t), C_{L}(t)\right)$, to capture dependence between the processes and their compensators. In the $\nu \rightarrow \infty$ limit these approach the bivariate normal distributions of $\left(Y_{1}(t), Y_{1}(t)-W_{1}(t)\right)$ and $\left(Y_{2}(t), \frac{1}{2}\left(Y_{1}(t)-W_{1}(t)\right)\right)$, respectively. Calculation of the covariance matrices is straightforward from our previous findings complemented by the formula

$$
\operatorname{Cov}\left(Y_{1}(t), W_{1}(t)\right)=-\sigma_{1}^{2}(1-t) \log (1-t)
$$

obtained by the Itó isometry. For instance

$$
\operatorname{Var}\left\{Y_{1}(t)-W_{1}(t)\right\}=\frac{4 \sqrt{2}}{3}\left(t-\frac{t^{2}}{2}+(1-t) \log (1-t)\right) .
$$

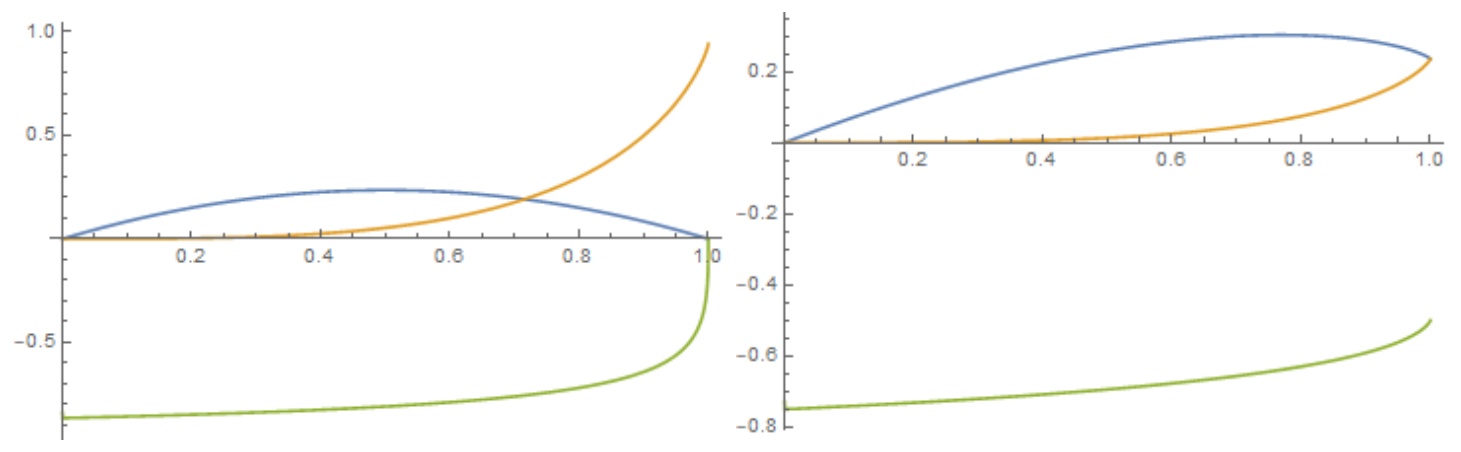

(a) $\operatorname{Var} Y_{1}(t), \operatorname{Var}\left\{Y_{1}(t)-W_{1}(t)\right\}$, $\operatorname{Corr}\left\{Y_{1}(t), Y_{1}(t)-W_{1}(t)\right\}$.

(b) $\operatorname{Var} Y_{2}(t), \operatorname{Var}\left\{\frac{1}{2}\left(Y_{1}(t)-W_{1}(t)\right\}, \quad\right.$, $\operatorname{Corr}\left\{Y_{2}(t), \frac{1}{2}\left(Y_{1}(t)-W_{1}(t)\right)\right\}$.

\section{Convergence of the moments}

The weak convergence shown in Theorem 4, combined with the convergence of moments for the majorant and minorant processes imply by virtue of 'Pratt's lemma' the expansion

$$
\mathbb{E}(X(t)-t)^{k}=\nu^{-k / 4} \mathbb{E} Y_{1}^{k}(t)+o\left(\nu^{-k / 4}\right), \quad k \in \mathbb{N},
$$

along with a similar expansion for the $t$-centred moment functions of $L / \sqrt{2 \nu}$. For $k=1$ the leading term vanishes, hence the convergence rate should be higher, as is evidenced in the instance $t=1$ by $(11)$. The logarithmic factor in (11) results from the optimality gap, hence it is of interest to inspect how the gap emerges in the course of selection.

We choose smallest possible constants $\beta_{-}>0, \beta_{+} \geq 0$ to squeeze the control function in the bounds

$$
\sqrt{\frac{2(1-x)}{\nu(1-t)}}-\frac{\beta_{-}}{\nu(1-t)} \leq \psi(t, x) \leq \sqrt{\frac{2(1-x)}{\nu(1-t)}}+\frac{\beta_{+}}{\nu(1-t)}, \quad t, x \in[0,1) .
$$


The condition $(22)$ thus holds with $\beta \geq \max \left(\beta_{-}, \beta_{+}, 1\right)$. To motivate introducing two parameters we note that for the optimal strategy (40) holds with $\beta_{+}=0$ ([8], Equation (3.5)), and that there is some asymmetry in the upper and lower estimates below.

The following auxiliary result is a special case of Grönwall inequality:

Lemma 5. Suppose function $f$ with $f(0)=0$ satisfies the integral inequality

$$
f(t) \leq-\int_{0}^{t} f(s)\left(\frac{1}{1-s}+\frac{a}{(1-s)^{2}}\right) \mathrm{d} s+\int_{0}^{t} g(s) \mathrm{d} s, \quad t \in[0,1),
$$

$a \in \mathbb{R}$. Then

$$
f(t) \leq(1-t) e^{-\frac{a}{1-t}} \int_{0}^{t} \frac{e^{\frac{a}{1-s}} g(s)}{1-s} \mathrm{~d} s, \quad t \in[0,1) .
$$

Proof. The linear operator defined by the right-hand side of (41) gives a solution to the associated integral equation. The assertion follows by observing that nonnegative $g$ is mapped to nonnegative $f$.

\subsection{Bounds on $p(t)$}

The upper bound in 40 implies

$$
\frac{\nu}{2} \psi^{2}(s, x)-1 \leq-\frac{x-s}{1-s}+\frac{\beta_{+} \sqrt{2}}{\sqrt{\nu}(1-s)} \sqrt{\frac{1-x}{1-s}}+\frac{\beta_{+}^{2}}{2 \nu(1-s)^{2}} .
$$

Using the elementary inequality $\sqrt{1-z} \leq 1-z / 2$ for $z \leq 1$ we obtain

$$
\mathbb{E} \sqrt{\frac{1-X(t)}{1-t}} \leq 1-\frac{\mathbb{E} X(t)-t}{2(1-t)} \leq 1-\frac{p(t)-t}{2(1-t)},
$$

and integrating in 42 yields

$$
p(t)-t \leq-\int_{0}^{t}(p(s)-s)\left(\frac{1}{1-s}+\frac{b}{(1-s)^{2}}\right) \mathrm{d} s+\int_{0}^{t} g(s) \mathrm{d} s,
$$

where

$$
g(t)=\frac{2 b}{(1-t)}+\frac{b^{2}}{(1-t)^{2}}, \quad b=\frac{\beta_{+}}{\sqrt{2 \nu}} .
$$

Applying Lemma 5 with $f(t)=p(t)-t$ and $a=b$ we obtain $p(t)-t \leq G(b, t)$, where

$$
G(b, t):=1+b-t-(1+b)(1-t) \exp \left(-\frac{b t}{1-t}\right) \text {. }
$$

For small $b>0$, this is a concave function, with $G(b, t)-b t$ changing sign from + to - at some point approaching $2 / 3$ as $b \rightarrow 0$. The asymptotic expansion

$$
G(b, t) \sim 2 t b+\frac{\left(2 t-3 t^{2}\right) b^{2}}{2(1-t)}, \quad b \rightarrow 0,
$$


holds uniformly, at least for $t$ bounded away from 1 ; therefore there is an upper bound $G(b, t)<2 b t+c_{+} b^{2}$, where the constant should be chosen to satisfy

$$
c_{+}>\max _{t \in[0,1]} \frac{2 t-3 t^{2}}{2(1-t)}=2-\sqrt{3} .
$$

It follows that

$$
p(t)-t \leq \frac{\beta_{+} \sqrt{2} t}{\sqrt{\nu}}+\frac{c_{+} \beta_{+}^{2}}{2 \nu},
$$

uniformly in $t \in[0,1]$ for sufficiently big $\nu$.

To estimate in the opposite direction, we have from the lemma $p(t)-t \geq G(b, t)$, this time with negative parameter

$$
b=-\frac{\beta_{-}}{\sqrt{2 \nu}} .
$$

Changing the variable to $T=(1-t)^{-1}$ simplifies analysis, and it is readily checked that

$$
T \mapsto T\left(G\left(b, 1-T^{-1}\right)-2 b\left(1-T^{-1}\right)-b^{2} T\right)
$$

is a concave function, positive in the range $1 \leq T<T_{0}$, where $T_{0}$ is such that $-b T_{0}$ approaches, as $|b| \rightarrow 0$, a limit value $1.7933 \ldots$ (the positive root of $1+x+x^{2}=e^{x}$ ), which we replace by smaller $\sqrt{2}$. Thus

$$
p(t)-t \geq-\frac{\beta_{-} \sqrt{2} t}{\sqrt{\nu}}-\frac{\beta_{-}^{2}}{2 \nu(1-t)}, \text { for } t \leq 1-\frac{\beta_{-}}{2 \sqrt{\nu}},
$$

provided $\nu$ is sufficiently large. But then by monotonicity from (44) it follows that

$$
p(t) \geq p\left(1-\frac{\beta_{-}}{2 \sqrt{\nu}}\right) \geq 1-\frac{\left(\sqrt{2}+\frac{3}{2}\right) \beta_{-}}{\sqrt{\nu}}, \quad \text { for } t>1-\frac{\beta_{-}}{2 \sqrt{\nu}},
$$

hence in this range of $t$

$$
p(t)-t \geq p(t)-1>-\frac{\left(\sqrt{2}+\frac{3}{2}\right) \beta_{-}}{\sqrt{\nu}},
$$

(also note that the trivial upper bound $p(t)-t<1-t<\frac{\beta_{-}}{2 \sqrt{\nu}}$ might improve upon 43 ) in this range).

Bounding the second term in (44) by its maximum, and combining with 46) into single inequality we obtain an estimate with simpler constant $3>\sqrt{2}+3 / 2$

$$
p(t)-t \geq-\frac{3 \beta_{-}}{\sqrt{\nu}}, \quad t \in[0,1]
$$

Similarly, the second term in 43 can be absorbed into the first with a larger constant. With the full range $t \in[0,1]$ covered, we have shown that

$$
\sup _{t \in[0,1]}|p(t)-t|=O\left(\frac{1}{\sqrt{\nu}}\right) \text {. }
$$




\subsection{Bounds on $q(t)$}

We turn to $q(t)=\mathbb{E} L(t) / \sqrt{2 \nu}$. For the upper estimate we use (7) to obtain an integral inequality

$$
\begin{aligned}
& q(t)=\mathbb{E} \int_{0}^{t} \sqrt{\nu / 2} \psi(s, X(s)) d s \leq \mathbb{E} \int_{0}^{t}\left(\sqrt{\frac{1-X(s)}{1-s}}+\frac{\beta_{+}}{\sqrt{2 \nu}(1-s)}\right) \mathrm{d} s \leq \\
& \int_{0}^{t}\left(1-\frac{p(s)-s}{2(1-s)}+\frac{\beta_{+}}{\sqrt{2 \nu}(1-s)}\right) \mathrm{d} s \leq \int_{0}^{t}\left(1-\frac{q(s)-s}{(1-s)}+\frac{\beta_{+}}{\sqrt{2 \nu}(1-s)}\right) \mathrm{d} s
\end{aligned}
$$

then apply Lemma 5 with $a=0$ to get

$$
q(t)-t \leq \frac{\beta_{+} t}{\sqrt{2 \nu}}
$$

The estimate approaches zero faster than in (11), but there is no disagreement since $q(1)<1$. Note that applying (7) and 43 straight incurs a second term.

For the optimal strategy, (40) holds with $\beta_{+}=0$, thus in this case $p(t)-t \leq 0$ and $q(t)-t \leq 0$.

Obtaining the lower bound is more challenging. Under the optimal strategy, the value function $F$ in (17) is concave [7], but under our more general assumptions on $\psi$ this need not be the case. However, by virtue of (21) we may replace $F$ by the concave function

$$
\widehat{F}(\nu):=\sqrt{2 \nu}-\frac{1}{12} \log (\nu+1)
$$

to obtain an expansion

$$
\mathbb{E} L(t)=\widehat{F}(\nu)-\mathbb{E}\{\widehat{F}(\nu(1-X(t))(1-t))\}+O(1),
$$

where the absolute value of the remainder is bounded uniformly in $t$ and $\nu$ by some constant $K$ only depending on $\beta_{-}$and $\beta_{+}$.

By monotonicity and concavity of $\widehat{F}$, using Jensen inequality and 47) we estimate

$$
\begin{gathered}
\mathbb{E}\{\widehat{F}(\nu(1-X(t))(1-t))\} \leq \widehat{F}(\nu(1-t)(1-p(t))= \\
\widehat{F}\left(\nu(1-t)^{2}\left(1-\frac{p(t)-t}{1-t}\right)\right) \leq \\
\widehat{F}\left(\nu(1-t)^{2}\left(1+\frac{3 \beta_{-}}{\sqrt{\nu}(1-t)}\right)\right)= \\
\sqrt{2 \nu}(1-t) \sqrt{1+\frac{3 \beta_{-}}{\sqrt{\nu}(1-t)}-} \frac{1}{12} \log \left\{\nu(1-t)^{2}\left(1+\frac{3 \beta_{-}}{\sqrt{\nu}(1-t)}\right)+1\right\}< \\
\left.\sqrt{2 \nu}(1-t)\left(1+\frac{3 \beta_{-}}{2 \sqrt{\nu}(1-t)}\right)-\frac{1}{12} \log \left(\nu(1-t)^{2}\right)\right)< \\
\sqrt{2 \nu}(1-t)+\frac{3 \beta_{-}}{\sqrt{2}}-\frac{1}{12} \log \nu-\frac{1}{6} \log (1-t) .
\end{gathered}
$$


Substituting this along with 49 into 50 we see that, for large enough $\nu$,

$$
\mathbb{E} L(t) \geq \sqrt{2 \nu} t+\frac{1}{6} \log (1-t)-\left(\frac{3 \beta_{-}}{\sqrt{2}}+K\right), \quad t \in[0,1] .
$$

The logarithmic term makes (51) useless for $t$ too close to 1 . However, cutting the range at, say $t_{0}:=1-1 / \sqrt{\nu}$, we can just employ the monotonicity to squeeze the expected length as

$$
F(\nu) \geq \mathbb{E} L(t) \geq \mathbb{E} L\left(t_{0}\right) \geq \sqrt{2 \nu}+\frac{1}{12} \log \nu-\left(\sqrt{2}+\frac{3 \beta_{-}}{\sqrt{2}}+K\right), \quad t \geq t_{0}
$$

For a better overview, we re-write 48 as

$$
\mathbb{E} L(t) \leq \sqrt{2 \nu} t+\beta_{+} t
$$

Comparing (51) with (52) it is seen that, uniformly in $t \in[0,1-h]$, the mean selected length $\mathbb{E} L(t)$ is within $O(1)$ from $\sqrt{2 \nu} t$, the latter being the exact mean length under the (unfeasible) stationary strategy.

With some more work we could show an upper bound with two leading terms as in (51) and a remainder uniformly bounded over $t<1-\nu^{-1 / 4+\epsilon}$.

\section{References}

[1] A. Arlotto, V.V. Nguyen and J.M. Steele (2015), Optimal online selection of a monotone subsequence: a central limit theorem, Stochastic Process. Appl. 125, 3596-3622.

[2] A. Arlotto, Y. Wei and X. Xie (2018), A O(log n)-optimal policy for the online selection of a monotone subsequence from a random sample, Random Structures and Algorithms 52, 41-53.

[3] S. Asmussen, Applied Probability and Queues, Springer, 2003.

[4] Y. M. Baryshnikov and A. V. Gnedin (2000), Sequential selection of an increasing sequence from a multidimensional random sample, Ann. Probab. 10(1), 258-267.

[5] A. Borovkov, Stochastic Processes in Queueing Theory, Springer, 1976.

[6] J. Brémaud, Point processes and Queues, Springer, 1981.

[7] F. T. Bruss and F. Delbaen (2001), Optimal rules for the sequential selection of monotone subsequences of maximum expected length, Stoch. Proc. Appl. 96, 313342.

[8] F. T. Bruss and F. Delbaen (2004), A Central Limit Theorem for the optimal selection process for monotone subsequences of maximum expected length, Stoch. Proc. Appl. 114, 287-311. 
[9] J. Bunge and C.M. Goldie (2001), Record sequences and their applications, Handbook of Statist. 19, 277-308.

[10] E.G. Coffman, L. Flatto and R.R. Weber (1987), Optimal selection of stochastic intervals under a sum constraint, Adv. Appl. Prob., 19, 454-473.

[11] D. Dauvergne, M. Nica and B. Virág (2019), Uniform convergence to the Airy line ensemble, ArXiv e-print 1907.10160

[12] P. S. Dey, M. Joseph and R. Peled (2018), Longest increasing path within the critical strip, ArXiv e-print 1808.08407

[13] A. Gnedin (1999), Sequential selection of an increasing subsequence from a sample of random size, Journal of Applied Probability 36(4), 1074-1085.

[14] A. Gnedin and A. Seksenbayev (2019), Asymptotics and renewal approximation in the online selection of increasing subsequence, ArXiv e-print 1904.11213

[15] E.V. Hmaladze (1981), Martingale approach in the theory of goodness-of-fit tests, Prob. Theory Appl. 26(2), 246-265.

[16] J. Jacod and A.N. Shiryaev, Limit Theorems for Stochastic Processes, SpringerVerlag, 1987.

[17] K. Johansson (1998), The longest increasing subsequence in a random permutation and a unitary random matrix model, Math. Res. Lett. 5(1-2), 63-82.

[18] O. Kallenberg, Foundations of Modern Probability, Springer, 2002.

[19] M. B. Marcus and J. Rosen, Markov processes, Gaussian processes and Local Times, CUP, 2006.

[20] D. Romik, The Surprising Mathematics of Longest Increasing Subsequences, CUP, 2015.

[21] S. M. Samuels and J.M. Steele (1981), Optimal sequential selection of a monotone sequence from a random sample, Ann. Probab. 9, 937-947. 